\section{PSICOLOGIA IBEROAMERICANA}

\section{Psicología Iberoamericana}

ISSN: 1405-0943

psicología.iberoamericana@uia.mx

Universidad Iberoamericana, Ciudad de

México

México

Del Castillo Arreola, Arturo; Guzmán Saldaña, Rebeca María Elena; Iglesias Hoyos, Scarlett; Reyes Lagunes, Isabel

Validez divergente y convergente del instrumento de autoeficacia al tratamiento en diabetes Psicología Iberoamericana, vol. 20, núm. 2, julio-diciembre, 2012, pp. 58-65

Universidad Iberoamericana, Ciudad de México

Distrito Federal, México

Disponible en: http://www.redalyc.org/articulo.oa?id=133928816008

Cómo citar el artículo

- Número completo

- Más información del artículo

- Página de la revista en redalyc.org

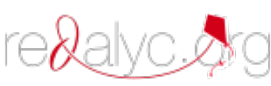

Sistema de Información Científica

Red de Revistas Científicas de América Latina, el Caribe, España y Portugal

Proyecto académico sin fines de lucro, desarrollado bajo la iniciativa de acceso abierto 


\title{
Validez divergente y convergente del instrumento de autoeficacia al tratamiento en diabetes
}

\author{
Divergent and Convergent Validity of the Diabetes Treatment \\ Self-efficacy Instrument
}

\author{
Arturo Del Castillo Arreola* \\ Rebeca María Elena Guzmán Saldaña \\ Scarlett Iglesias Hoyos \\ Universidad Autónoma del Estado de Hidalgo \\ Isabel Reyes Lagunes \\ Universidad Nacional Autónoma de México
}

\section{RESUMEN}

Factores psicosociales, como la percepción de autoeficacia para seguir el tratamiento, han mostrado ser importantes predictores de las conductas de autocuidado de pacientes con diabetes tipo 2. El Instrumento de Autoeficacia al tratamiento en diabetes ha mostrado tener características psicométricas adecuadas para medir esta variable en México; sin embargo, no se cuentan con datos de validez convergente y divergente de la prueba. Para realizar esta validación participaron 240 pacientes con diagnóstico confirmado de diabetes tipo 2, seleccionados de forma intencional en centros de salud del estado de Hidalgo. Los resultados del análisis de correlación de Pearson indican relaciones positivas entre el nivel de autoeficacia y de bienestar psicológico, y correlaciones negativas con los niveles de malestar emocional y hemoglobina glucosilada. La escala validada podrá ser utilizada en el monitoreo de los niveles de autoeficacia al tratamiento en pacientes con diabetes en clínicas del primer nivel de atención en salud de nuestro país.

Palabras clave: autoeficacia, instrumento, validez convergente, validez divergente, diabetes.

\section{ABSTRACT}

Psychosocial factors as treatment self-efficacy have shown to be important predictors of diabetes self-management behaviors. The Diabetes Treatment Self-efficacy Instrument have shown proper psychometric characteristics to measure this variable in Mexico, nevertheless, it doesn't exist convergent and divergent validity data of this questionnaire. In order to make this validation, an intentional sample of 240 patients with type 2 diabetes were invited at Hidalgo's health centers. Pearson correlation coefficient results indicate positive relations between self-efficacy treatment and psychological well being levels; and negative relations between self-efficacy, distress and glycated hemoglobin levels. The validated scale may be used in the monitoring of self-efficacy treatment levels with diabetes patients at first level health attention clinics of our country.

Keywords: self-efficacy, instrument, convergent validity, divergent validity, diabetes

\footnotetext{
* Para cualquier comentario sobre el presente artículo comunicarse con: Arturo Del Castillo Arreola. 7717172000 Ext. 5104, artur78@yahoo.com Rebeca María Elena Guzmán Saldaña. 7717172000 Ext. 5104, remar64@yahoo.com.mx Scarlett Iglesias Hoyos. 7717172000 Ext. 5104, scarlett_iglesias@hotmail.com Isabel Reyes Lagunes. 55 56322323, lisabel@servidor.unam.mx
} 


\section{INTRODUCCIÓN}

Dentro del grupo de padecimientos crónico-degenerativos cuya prevalencia ha aumentado de forma alarmante las últimas décadas se encuentra la diabetes (Secretaría de Salud [ssa], 2010). Se calcula que en el mundo hay aproximadamente 366 millones de personas con esta condición y se estima que esta cifra aumente a 552 millones para el año 2030 (Federación Internacional de Diabetes [FID], 2011).

México ocupa el séptimo lugar a nivel mundial entre los países con un mayor número de pacientes con diabetes, con alrededor de 10.3 millones de personas, y se estima que para el 2030 ocupe el sexto lugar, con 16.4 millones de personas (FID, 2011).

De los distintos tipos de diabetes que existen, del 90 al 95\% de los pacientes padecen diabetes tipo 2. Este tipo de diabetes (antes denominada diabetes mellitus no insulino dependiente (DMNID), diabetes tipo II o diabetes del adulto) es aquella que se presenta en individuos que tienen resistencia a la insulina y habitualmente una deficiencia relativa (en lugar de absoluta) de ésta (ADA, 2012a).

Para el tratamiento de la enfermedad resulta fundamental que el paciente lleve a cabo modificaciones en su conducta, como: 1) seguir un plan alimenticio personalizado y cuidar su peso corporal, 2) auto monitorear sus niveles de glucosa en sangre y/o en orina, 3) llevar a cabo actividades físicas continuamente, 4) atender a las indicaciones farmacológicas correspondientes, y 5) realizar visitas a diferentes especialistas de la salud (ADA, 2012b). Este tratamiento es relativamente complejo, prolongado y requiere de disciplina, planeación y adaptación a cambios que los pacientes y sus familias no siempre están preparados para desempeñar (Hotz, Kaptein, Pruitt, Sánchez-Sosa \& Willey, 2003).

\section{Autoeficacia al tratamiento en diabetes}

Dentro del grupo de factores psicosociales relacionados con el manejo de la enfermedad, las creencias de autoeficacia hacia el tratamiento intervienen de manera significativa en el automanejo y los resultados de pacientes con diabetes (Del Castillo \& Martínez, 2010). El constructo de autoeficacia fue introducido por Bandura (1997) y representa un aspecto nuclear de la teoría social cognoscitiva. De acuerdo con esta teoría, la motivación humana y la conducta están re- gulados por el pensamiento e involucran tres tipos de expectativas: a) aquéllas relacionadas con la situación, en la que las consecuencias son producidas por eventos ambientales independientes de la acción personal, b) las de resultado, que se refieren a las creencias de que una conducta producirá determinados resultados, y por último, c) las expectativas de autoeficacia o autoeficacia percibida, que son las creencias que tiene una persona de poseer las capacidades para desempeñar las acciones necesarias que le permitan obtener los resultados deseados.

Los niveles de autoeficacia determinan la motivación. Una vez que se ha iniciado un curso de acción, las personas con alta autoeficacia invierten más esfuerzo, son persistentes y mantienen mayor compromiso con sus metas frente a las dificultades (Bandura, 1997). Las investigaciones revelan que altos niveles de autoeficacia tienen consecuencias beneficiosas para el funcionamiento del individuo y su bienestar general (KleinHessling, Lohaus \& Ball, 2005).

Con relación a las conductas preventivas en salud, los individuos con alta autoeficacia percibida tienen más probabilidad de iniciar cuidados preventivos, buscar tratamientos tempranos y ser más optimistas con respecto a la eficacia de éstos (Grembowski, Patrick, Diehr, Durham, Beresford et al., 1993). Además, las personas con alta autoeficacia percibida también tienen mayor probabilidad de recuperarse mejor y más rápidamente de las enfermedades que quienes tienen baja autoeficacia (Grembowski et al., 1993).

Debido a que el automanejo de la diabetes involucra una serie de factores conductuales, personales y ambientales que interactúan en la realización de las actividades cotidianas de autocuidado, se han asociado altos niveles de autoeficacia con un adecuado automanejo en diabetes en adolescentes (Littlefield, Craven, Rodin, Daneman, Murray et al., 1992); y adultos con diabetes tipo 1 y tipo 2 (Aljasem, Peyrot, Wissow \& Rubin, 2001; Bernal, Woolley, Schenaul \& Dickinson, 2000; King et al., 2010).

Con respecto a la evaluación de la autoeficacia en personas con diabetes, se han diseñado diversos instrumentos de medición, muchos enfocados a pacientes con diabetes tipo 1, como la Escala de Autocuidado en Diabetes (Confidence in Diabetes Self-Care Scale) (Van der Ven, Adér, Weinger, Van der Ploeg, Yi et al., 2000), que 
es un instrumento de autorreporte de 20 reactivos, los cuales evalúan aspectos como la autoeficacia y la habilidad percibida para desempeñar tareas de autocuidado.

Entre las escalas construidas para pacientes con diabetes tipo 2 se encuentra el Cuestionario para la Autoeficacia en Diabetes (DSEQ, por sus siglas en inglés), desarrollado por Robin, Little y McGuire (2004) con el propósito de evaluar dos aspectos generales de este constructo: las creencias acerca de la importancia de seguir tareas de autocuidado y la confianza en la habilidad para realizar dichas tareas. También se encuentra el Cuestionario Multidimensional de Diabetes (Talbot, Nouwen, Gingras, Goselin \& Audet, 1997), que evalúa aspectos sociales y cognoscitivos relacionados con la diabetes, y está integrado por tres subescalas: 1) percepciones de la diabetes y apoyo social; 2) reforzamiento de conductas positivas y erróneas relacionadas con actividades de autocuidado; 3) autoeficacia y expectativas de resultados relacionadas con el tratamiento de la diabetes.

Por último, la Escala de Empoderamiento en Diabetes (Diabetes Empowerment Scale), es un instrumento elaborado por el Michigan Diabetes Research and Training Center (MDRTC, 2008) que representa una medida válida y confiable para evaluar la autoeficacia relacionada con el cuidado de la diabetes en diversos contextos.

\section{Instrumento de autoeficacia al tratamiento en diabetes}

Si bien es cierto que se han diseñado múltiples instrumentos que evalúan la autoeficacia en personas con diabetes tipo 1 y tipo 2, éstos, por lo general, no dirigen sus reactivos hacia conductas específicas como la realización de actividad física, toma adecuada de medicamentos y seguimiento del plan alimenticio; además de que no existía una escala con las características mencionadas que hubiera sido estandarizada en población mexicana. De ahí que en un estudio previo, Del Castillo (2010) llevó a cabo la construcción de Instrumento de Autoeficacia al Tratamiento en pacientes con diabetes tipo 2 en México.

El instrumento quedó conformado por 14 reactivos distribuidos en los siguientes factores: 1) autoeficacia en el seguimiento del plan alimenticio (seis reactivos); 2) autoeficacia en la realización de activi- dad física (cinco reactivos) y 3 ) autoeficacia en la toma de medicamentos orales (tres reactivos). Se realizó un análisis alpha de Cronbach para determinar la consistencia interna de la escala en su totalidad, así como las tres subescalas resultantes. La escala total tuvo un índice alpha de .83, mientras que los índices alpha de las subescalas fueron los siguientes: 1) autoeficacia en el seguimiento del plan alimenticio .78; 2) autoeficacia en la realización de actividad física .80 ; y 3 ) autoeficacia en la toma de medicamentos orales 63 .

Al tener como antecedentes los datos de evidencias de validez de constructo obtenidos mediante la identificación de las dimensiones del instrumento, el propósito del presente estudio consistió en mostrar evidencias de validez de constructo convergente y divergente del Instrumento de Autoeficacia al tratamiento en pacientes con diabetes.

Para alcanzar el objetivo antes señalado se midieron variables como el malestar emocional asociado a la diabetes y el nivel de hemoglobina glucosilada que teóricamente divergen con el constructo autoeficacia al tratamiento. También se midió el nivel de bienestar psicológico, variable que converge teóricamente con el constructo en cuestión.

Se esperaban correlaciones moderadas y negativas (-.30 a -.50) entre el nivel de autoeficacia al tratamiento en diabetes, el nivel de hemoglobina glucosilada y el de malestar emocional asociado a diabetes de la muestra en estudio. Por otro lado se esperaban correlaciones moderadas y positivas (.30 a .50) entre el nivel de autoeficacia y el nivel de bienestar psicológico de los pacientes seleccionados.

\section{MÉTODO}

\section{Sujetos}

En el estudio participaron 240 pacientes con diagnóstico confirmado de diabetes tipo 2 seleccionados de forma intencional en centros de salud del estado de Hidalgo. De la muestra total, 63 (26.3\%) eran hombres y $177(73.8 \%)$ mujeres. Con respecto a la escolaridad, 181 (75.4\%) de los participantes contaban con primaria, $35(14.6 \%)$ con secundaria, 18 (7.5\%) preparatoria y $6(2.5 \%)$ con licenciatura.

En la distribución de los participantes con respecto a la ocupación, 125 (52.1\%) eran amas de casa, 48 
(20\%) contaban con un trabajo de tiempo completo, 38 (15.8\%) un trabajo de medio tiempo, 11 (4.6\%) presentaban una discapacidad que les impedía trabajar, 10 (4.2\%) eran desempleados y 6 (2.5\%) estaban retirados. Por lo que respecta al estado civil, 158 (65.8\%) de los participantes estaban casados o vivían en unión libre, mientras que 82 (34.2\%) vivían solos. En cuanto a los años de diagnóstico, 22 (9.2\%) de los participantes tenían menos de un año de diagnóstico, 81 (33.8\%) habían sido diagnosticados de dos a cinco años antes de la evaluación, 62 (25.8\%) tenían entre seis y 10 años de diagnóstico, mientras que 75 (31.3\%) de ellos contaban con más de 11 años.

De acuerdo con el tipo de tratamiento, la muestra estuvo distribuida de la siguiente manera: 187 (77.9\%) sólo tomaban hipoglucemiantes orales, 47 (19.6\%) combinaban hipoglucemiantes orales e insulina, 4 (1.7\%) utilizaba únicamente insulina, mientras que $2(.8 \%)$ tenía como tratamiento únicamente un plan alimenticio.

\section{Instrumentos}

Bienestar psicológico: Se midió utilizando la versión adaptada y validada en población mexicana con diabetes del Índice de Bienestar Psicológico OMS-5 (WHO-5 Well-Being Index) (Del Castillo, Rodríguez, Martínez, Guzmán y Reyes-Lagunes, 2008).

Este instrumento se diseñó para evaluar la dimensión de bienestar psicológico que considera muchos de los aspectos subjetivos de la calidad de vida, más allá de los aspectos objetivos, que se circunscriben a la evaluación de la capacidad o incapacidad de los pacientes para llevar a cabo sus actividades diarias (Bech, Olsen, Kajoller \& Rasmussen, 2003). El OMS-5 es una escala autoaplicable, originalmente redactada en idioma danés, compuesta por cinco reactivos redactados en forma positiva con seis opciones de respuesta que van de 0 , nunca, a 5 , todo el tiempo. Los reactivos se distribuyen en tres áreas: humor positivo (buen humor, relajado), vitalidad (estar activo y despertar descansado) e interés general (estar interesado en las cosas). Se ha utilizado con mucho éxito como prueba de detección de depresión en centros de primer nivel de atención en salud en distintos estudios y ha mostrado altos índices de sensibilidad y especificidad en relación con otros instrumentos que también detectan depresión (Henkel et al., 2003). La adaptación del instrumento en pobla- ción mexicana mantiene la conformación de un solo factor y muestra datos de consistencia interna por la fórmula alpha de Cronbach de .82 (Del Castillo et al., 2008).

Control metabólico: Se evaluó mediante el índice de Hemoglobina Glucosilada (HbA1c) que corresponde al promedio en el nivel de glucosa en los tres meses anteriores a la toma de la muestra (ADA, 2012a).

Malestar emocional asociado a diabetes: Se evaluó con la versión adaptada en población mexicana del Cuestionario de Áreas Problema en Diabetes-PAID (Del Castillo \& Reyes- Lagunes, 2010). El instrumento consta de 16 reactivos distribuidos en tres factores: 1) emociones negativas (siete reactivos), 2) problemas relacionados con el tratamiento (seis reactivos) y 3 ) problemas relacionados con el apoyo (tres reactivos). Es una escala tipo Likert que presenta reactivos relacionados con eventos relacionados con el manejo de la diabetes y cuenta con cinco opciones de respuesta que van de 1, "no es un problema", a 5, "es un problema grave". La escala total presenta un índice de consistencia interna de Cronbach de .90 .

\section{Procedimiento}

Para conocer la validez convergente y divergente del Instrumento de Autoeficacia al Tratamiento en Diabetes se llevaron a cabo correlaciones producto momento de Pearson entre la escala total y subescalas de la prueba antes señalada, el Índice de Bienestar Psicológico OMS-5, el indicador de Hemoglobina Glucosilada HbA1c y el Cuestionario de Áreas Problema en Diabetes PAID.

\section{RESULTADOS}

En la tabla 1 se muestran los resultados del análisis de correlación de Pearson para conocer las relaciones entre las escalas y subescalas del Instrumento de Autoeficacia en Diabetes con las escalas y subescalas del Cuestionario de Áreas Problema en Diabetes (PAID), el Instrumento de Bienestar Psicológio (OMS) y el nivel de control metabólico medido con el índice de hemoglobina glucosilada HbA1c de los pacientes.

Como se observa en la tabla 1, existen relaciones estadísticamente significativas entre el nivel de autoeficacia total con el nivel de hemoglobina glucosilada, el nivel de bienestar psicológico medido con el OMS-5, así 
Tabla 1. Correlaciones del Instrumento de Autoeficacia al Tratamiento en Diabetes y sus subescalas con otras variables de efecto

\begin{tabular}{|l|c|c|c|c|c|c|}
\hline & HbA1c & OMS- 5 & PAID Total & $\begin{array}{c}\text { PAID } \\
\text { Emociones }\end{array}$ & $\begin{array}{c}\text { PAID } \\
\text { Tratamiento }\end{array}$ & PAID Apoyo \\
\hline $\begin{array}{l}\text { Autoeficacia } \\
\text { Total }\end{array}$ & $-.19^{*}$ & $.32^{*}$ & $-.42^{*}$ & $-.31^{*}$ & $-.43^{*}$ & $-.33^{*}$ \\
\hline $\begin{array}{l}\text { Autoeficacia } \\
\text { Alimentación }\end{array}$ & $-.24^{*}$ & $.33^{*}$ & $-.39^{*}$ & $-.29^{*}$ & $-.40^{*}$ & $-.29^{*}$ \\
\hline $\begin{array}{l}\text { Autoeficacia } \\
\text { Ejercicio }\end{array}$ & -.05 & $.27^{*}$ & $-.29^{*}$ & $-.22^{*}$ & $-.28^{*}$ & $-.24^{*}$ \\
\hline $\begin{array}{l}\text { Autoeficacia } \\
\text { Medicameno }\end{array}$ & $-.21^{*}$ & .10 & -.16 & $-.01^{*}$ & $-.28^{*}$ & $-.16^{*}$ \\
\hline
\end{tabular}

Nota. ${ }^{*}$ Correlaciones significativas $\mathrm{p}<.01$

Tabla 2. Coeficientes estandarizados de regresión lineal simple teniendo como variable independiente el nivel de autoeficacia total del instrumento

\begin{tabular}{|l|c|c|c|c|}
\hline & R2 & B & t & Sig. \\
\hline HbA1C & .036 & -.190 & -1.899 & .061 \\
\hline OMS-5 & .108 & .328 & 3.756 & .000 \\
\hline PAID Total & .183 & -.428 & -5.117 & .000 \\
\hline
\end{tabular}

Nota. ${ }^{*} \mathrm{p}<.01$

como con el nivel de malestar emocional total medido con el PAID. Se muestra que a mayor nivel de autoeficacia al tratamiento encontraremos niveles mayores de bienestar psicológico y niveles menores de hemoglobina glocosilada y malestar emocional en la muestra estudiada.

También se realizó análisis de regresión lineal simple para conocer si el nivel total de autoeficacia al tratamiento cuenta con capacidad predictiva sobre los niveles totales del instrumento de bienestar psicológico y el nivel de hemoglobina glucosilada.

De acuerdo con los datos que se muestran en la tabla 2, el nivel de autoeficacia al tratamiento predice el $10 \%$ de la varianza explicada del bienestar psicológico y el $18 \%$ de la varianza del malestar emocional asociado a diabetes en la muestra de estudio; cabe mencionar que estos resultados son estadísticamente significativos.

\section{DISCUSIÓN}

Se llevó a cabo análisis de asociación y predictivos para dar muestras de validez convergente y divergen- te del Instrumento de Autoeficacia al Tratamiento en Diabetes. Para alcanzar el objetivo antes señalado se realizaron análisis de correlación de Pearson y análisis de regresión lineal simple, incluyendo los niveles de autoeficacia, malestar emocional, bienestar psicológico y hemoglobina glucosilada de una muestra de pacientes con diabetes.

Con respecto a los datos de validez convergente se encontró que mientras mayor sea el nivel de autoeficacia al tratamiento, mayor será el nivel de bienestar psicológico de los pacientes con diabetes. Las correlaciones positivas estadísticamente significativas entre ambas variables se mantienen en las escalas de autoeficacia al plan alimenticio y autoeficacia en la realización de actividad física. Por lo que respecta al análisis predictivo, el nivel de autoeficacia al tratamiento explica un porcentaje moderado del bienestar psicológico de estos pacientes.

Estos datos muestran convergencia entre las dos medidas y añaden información a los resultados encontrados en otros estudios, que demuestran que altos ni- 
veles de autoeficacia tienen consecuencias beneficiosas para el funcionamiento del individuo y su bienestar general (Klein-Hessling, Lohaus \& Ball, 2005).

Además, los estudios indican que los individuos con alta autoeficacia percibida también tienen más probabilidad de evaluar su salud como mejor, estar menos enfermos o depresivos, y recuperarse mejor y más rápidamente de las enfermedades, que las personas con baja autoeficacia (Grembowski et al., 1993).

Asimismo, mayores niveles de bienestar psicológico y de autoeficacia al tratamiento incidirán en la motivación del paciente, aumentando la probabilidad de que invierta mayor esfuerzo en llevar a cabo las conductas de tratamiento, que sea más persistente y mantenga mayor compromiso con sus metas frente a las dificultades (Bandura, 1997).

La validez divergente del instrumento se exploró correlacionando la escala total y cada una de las subescalas del PAID con el Instrumento de Autoeficacia en Diabetes y sus diferentes dimensiones. Se encontraron relaciones estadísticamente significativas negativas entre los puntajes de ambos instrumentos en cada una de sus subescalas, a excepción de la de autoeficacia al medicamento. Así, a mayor nivel de malestar emocional hubieron niveles menores de autoeficacia al tratamiento total, así como en las subescalas que miden la autoeficacia al seguimiento del plan alimenticio y a la realización de actividad física.

Estos datos coinciden con investigaciones previas que señalan la relación entre altos niveles de malestar emocional y bajos niveles de autoeficacia para llevar a cabo las conductas de autocuidado en pacientes con distintos problemas crónicos (Lloyd, Wing, Orchard \& Becker, 1993). Las reacciones emocionales que el paciente experimenta ante estresores externos pueden generar cambios en el nivel de autoeficacia al tratamiento, lo que posteriormente derivará en menores niveles de actividad física, menor seguimiento del plan alimenticio y de la toma de medicamentos (Albright, Parchman \& Burge, 2001; Lin, Katon, Von, Korff, Rutter et al., 2004).

También se exploró la validez divergente del Instrumento de Autoeficacia al tratamiento al correlacionar los puntajes de la escala total y sus subescalas con el nivel de control metabólico de los pacientes que conformaron la muestra estudiada. Se utilizó el nivel de hemoglobina glucosilada como indicador de control metabólico del promedio de glucosa en sangre de los pacientes durante los tres meses anteriores a la medición. Los resultados indican que a mayor nivel de autoeficacia en diabetes menor será el nivel de hemoglobina glucosilada de los pacientes. Las correlaciones negativas se mantienen en las subescalas del instrumento de autoeficacia al tratamiento, con excepción de la subescala de autoeficacia a la realización de actividad física.

Por lo que respecta al análisis predictivo, el nivel de autoeficacia al tratamiento explica un porcentaje moderado del malestar emocional de los pacientes.

Estos resultados coinciden con investigaciones previas que han mostrado que debido a que el automanejo de la diabetes involucra una serie de factores conductuales, personales y ambientales que interactúan en la realización de las actividades cotidianas de autocuidado, altos niveles de autoeficacia se asocian con un adecuado automanejo en diabetes (Aljasem, Peyrot, Wissow \& Rubin, 2001; King et al., 2010).

Ahora se cuenta con una medida confiable y válida para medir la autoeficacia al tratamiento en pacientes con diabetes tipo 2 en México. La mayor parte de los instrumentos diseñados para medir esta variable se han desarrollado en otros países y en pacientes con diabetes tipo 1 (Van der Ven et al., 2000). De aquellos enfocados a la evaluación de pacientes con diabetes tipo 2, en su mayoría no abarcan la gama de conductas específicas que estos pacientes suelen llevar a cabo para tener un adecuado control de la enfermedad (MDRTC, 2008; Robin et al., 2004; Talbot et al., 1997).

Los resultados del presente estudio muestran una adecuada validez convergente y divergente del Instrumento de Autoeficacia al Tratamiento en Diabetes. Esta prueba es un instrumento de breve aplicación que podrá utilizarse en la consulta médica para identificar el nivel de autoeficacia al tratamiento en pacientes con diabetes tipo 2, lo que permitirá tomar decisiones adecuadas para el manejo del problema y desarrollar intervenciones psicológicas exitosas que ayuden al paciente a tener un adecuado control metabólico y una mejor calidad de vida. 


\section{REFERENCIAS}

Albright, T.L., Parchman, M. \& Burge, S.K. (2001). The RRNeST Investigators: Predictors of self-care behavior in adults with type 2 diabetes: an RRNeSTStudy. Family Medicine Journal, 33, 354-360.

Aljasem, L., Peyrot, M., Wissow, L. \& Rubin, R. (2001). The impact of barriers and self-efficacy on self-care behaviors in type 2 diabetes. Diabetes Educator, 27, 393-404.

American Diabetes Association (2012a). Diagnosis and Classification of Diabetes Mellitus. Diabetes Care, 35, sup1, s64-s71. doi:10.2337/dc12-s064.

American Diabetes Association (2012b). Standards of Medical Care in Diabetes-2012. Diabetes Care, 35, sup1, s11-s63. doi:10.2337/dc12-s011

Bandura, A. (1997). Self-Efficacy: The Exercise of Control. New York: Freeman.

Bech, P., Olsen, L.R., Kajoller, M. \& Rasmussen, N.K. (2003). Measuring well-being rather than the absence of distress symptoms: a comparison of the SF-36 Mental Health Subscale and the WHO-Five Well Being Scale. International Journal of Methods and Psychiatry Research, 12, 2, 85-91.

Bernal, H., Woolley, S., Schenaul, J. \& Dickinson, J. (2000). Correlates of self-efficacy in diabetes selfcare among Hispanic adults with diabetes. Diabetes Educator, 26, 673-680.

Del Castillo, A. (2010). Apoyo social, síntomas depresivos, autoeficacia y bienestar psicológico en pacientes con diabetes tipo 2. Tesis de Doctorado. Universidad Nacional Autónoma de México.

Del Castillo, A. \& Martínez, J. (2010). Disfunción sexual en hombres y mujeres con diabetes. En J. Morales, E. Madrigal, G. Nava, I. Durante, A. Jonguitud \& J. Esquivel (Eds.) Diabetes. (Segunda Ed.). Universidad Autónoma del Estado de Hidalgo.

Del Castillo, A. \& Reyes-Lagunes, I. (2010). Validación convergente y divergente del Cuestionario de Áreas Problema en Diabetes. En M. García, A. Del Castillo, R. Guzmán \& J. Martínez (Eds.) Medición en Psicología: del individuo a la interacción. México: Universidad Autónoma del Estado de Hidalgo.

Del Castillo, A., Rodríguez, J., Martínez, J.P., Guzmán, R. \& Reyes-Lagunes, I. (2008). Adaptación del Bienestar Psicológico de la OMS-5 en pacientes mexicanos con diabetes tipo 2. En S. Rivera, R. Díaz-Loving,
R. Sánchez \& I. Reyes-Lagunes (Eds.) La Psicología Social en México. XII. 55-59.

Federación Internacional de Diabetes (2011). Diabetes Atlas. (Quinta Ed.). Bélgica: Federación Internacional de Diabetes.

Federación Mexicana de Diabetes. (2010). Diabetes en números. Recuperado el 8 de abril de 2010, de www. fmdiabetes.org/v2/paginas/d-numerosphp

Grembowski, D., Patrick, D., Diehr, P., Durham, M., Beresford, S., Kay, E. \& Hecht, J. (1993). Self-efficacy and behavior among older adults. Journal of Health and Social Behavior, 34, 89-104.

Henkel, V., Mergl, R., Kohnen, R., Maier, W., Möller, H. \& Hegerl, U. (2003). Identifying depression in primary care: a comparison of different methods in a prospective cohort study. British Medical Journal, 326, 200-201.

Hotz, S., Kaptein, A., Pruitt, S., Sánchez-Sosa, J. J. \& Willey, C. (2003). Behavioral mechanisms explaining adherence: What every health professional should know. In WHO (Eds.) Adherence to long term therapies: Evidence for action (pp. 135-149). Geneva: World Health Organization.

King, D., Glasgow, R., Toobert, D., Strycker, L., Estabrooks, P., Osuna, D. \& Faber, A. (2010). Self-Efficacy, Problem Solving, and Social-Environmental Support Are Associated With Diabetes Self-Management Behaviors. Diabetes Care, 33(4), 751-753.

Klein-Hessling, J., Lohaus, A. \& Ball, J. (2005). Psychological predictors of health-related behavior in children. Psychology, Health \& Medicine, 10(1), 31-43.

Lin, E.H., Katon, W., Von, Korff M., Rutter, C., Simon, G.E., Oliver, M., Ciechanowski, P., Ludman, E.J., Bush, T. \& Young, B. (2004). Relationship of depression and diabetes self-care, medication adherence, and preventive care. Diabetes Care, 27, 2154-2160.

Littlefield, C.H., Craven, J.L., Rodin, G.M., Daneman, D., Murray, M.A. \& Rydall, A.C. (1992). Relationship of self-efficacy and binging to adherence to diabetes regimen among adolescents. Diabetes Care, 15, 90-94.

Lloyd, C. E, Wing, R. R, Orchard, T. J \& Becker D. J. (1993). Psychosocial correlates of glycemic control: the Pittsburgh Epidemiology of Diabetes Complications (EDC) Study. Diabetes Research and Clinical Practice, 21, 187-195. 
Michigan Diabetes Research and Training Center (MDRTC) (2008). Diabetes Empowerment Scale (DES). Recuperado el 30 de abril de 2008 de http:// www.measurementexperts.org/instrument_reviews. asp?detail $=71$

Robin, N. Little, M. \& McGuire, H. (2004). Diabetes SelfEfficacy Questionnaire (DSEQ). Journal of Health Psychology, 12, 5-33.

Secretaría de Salud de Hidalgo. (2010). Actualización del programa de programa estatal de salud. Hidalgo: ssH.
Talbot F, Nouwen A, Gingras J, Gosselin M. \& Audet J. (1997). The assessment of diabetes related cognitive and social factors: the Multidimensional Diabetes Questionnaire. Journal of Behavioral Medicine, 20(3), 291-312.

Van der Ven, N., Adèr, H., Weinger, K., Van der Ploeg, Yi, J., Snoek, F. \& Pouwer, F. (2000). The Confidence in Diabetes Self-Care Scale. Psychometric properties of a new measure of diabetes-specific self-efficacy in Dutch and U.S. patients with type 1 diabetes. Diabetes Care, 21, 41-67. 